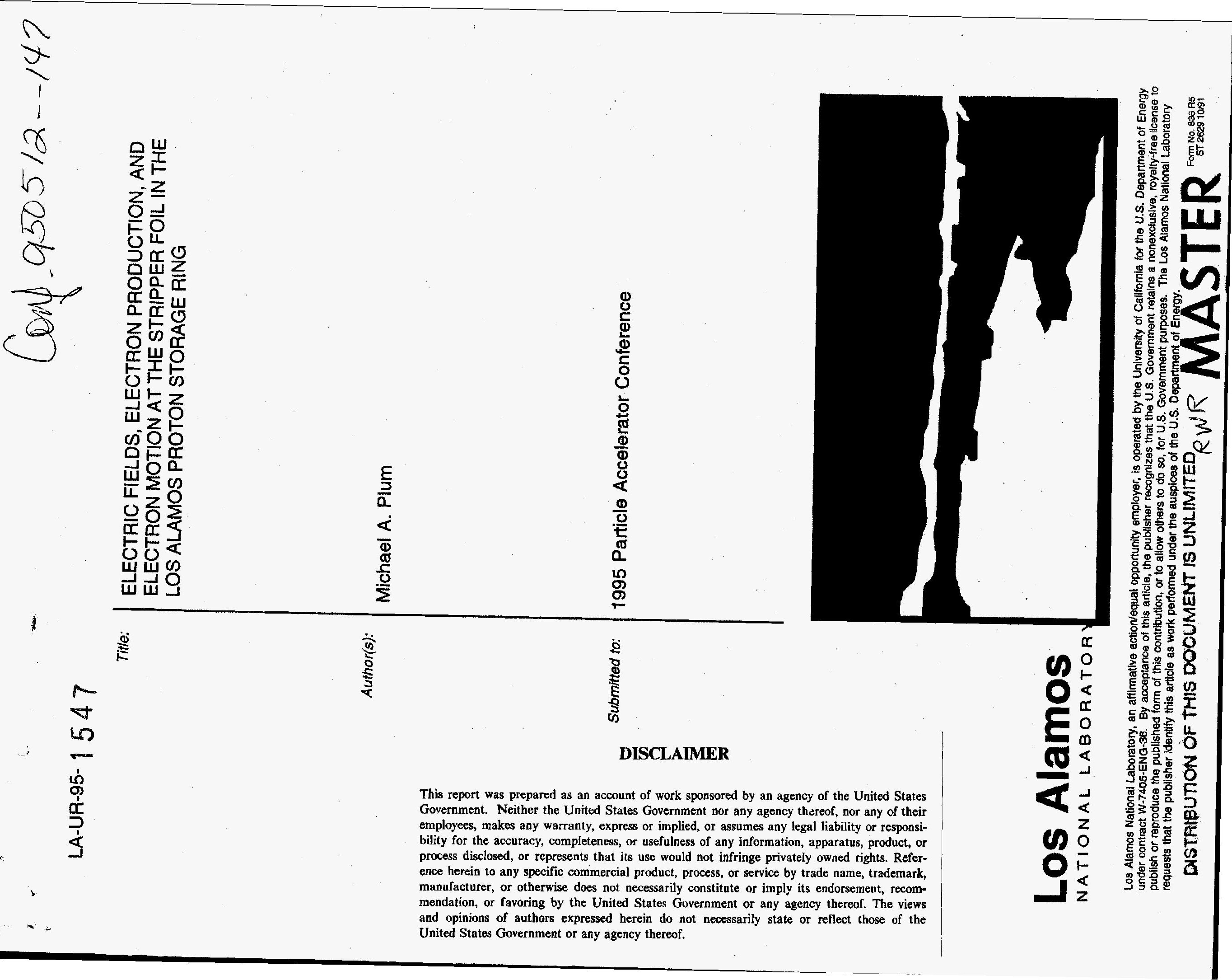




\section{DISCLAIMER}

Portions of this document may be illegible in electronic image products. Images are produced from the best available original document. 


\title{
ELECTRIC FIELDS, ELECTRON PRODUCTION, AND ELECTRON MOTION AT THE STRIPPER FOIL IN THE LOS ALAMOS PROTON STORAGE RING
}

\author{
M. PLUM \\ Accelerator Operations and Technology Division \\ Los Alamos National Laboratory, Los Alamos, NM 87545
}

The beam instability at the Los Alamos Proton Storage Ring (PSR) most likely involves coupled oscillations between electrons and protons. For this instability to occur, we must have a strong source of electrons, and so we have begun to investigate the various sources of electrons in the PSR. We expect copious electron production in the injection section because this section contains the stripper foil. This foil is mounted near the center of the beam pipe, and both circulating and injected protons pass through it, thus allowing ample opportunity for electron production. In this paper we will discuss various mechanisms for electron production, beaminduced electric fields, and electron motion in the vicinity of the foil.

\section{INTRODUCTION}

In the PSR, the stripper foil is a $200 \mu \mathrm{g} / \mathrm{cm}^{2}$ carbon foil suspended near the center of the beam pipe by a web of carbon fibers. Many electrons are created when both the circulating and injected protons pass through this foil, as shown in Table 1. We have identified four sources: "convoy" electrons stripped from the injected $\mathrm{H}^{\circ}$ beam, secondary-emission (SEM) electrons due to the injected and circulating particles passing through the foil, thermionic electrons due to foil heating, and delta-ray (knock-on) electrons due to the injected and circulating protons passing through the foil. We also expect SEM from any beam-pipe surfaces the beam may interact with, and electron-ion pairs from residual-gas ionization.

\section{ELECTRON PRODUCTION}

The convoy electron-production rate is the same as the incoming $\mathrm{H}^{\circ}$ particle rate, minus the $7 \%$ to $10 \%$ stripping inefficiency. During normal production conditions, the peak

Table 1. Electron production per injected $\mathrm{H}^{0}$ particle.

\begin{tabular}{|l|l|l|l|}
\hline Electron source & $\begin{array}{l}\text { no. per } \\
\text { injected } \\
\mathbf{H}^{6}\end{array}$ & $\begin{array}{l}\text { Kinetic. } \\
\text { Energy. }\end{array}$ & $\begin{array}{l}\text { Average } \\
\text { Current }\end{array}$ \\
\hline Convoy electrons & 1.0 & $430 \mathrm{keV}$ & $75 \mu \mathrm{A}$ \\
\hline Secondary electrons & 3.6 & $\begin{array}{l}\text { up to } \\
-20 \mathrm{eV}\end{array}$ & $270 \mu \mathrm{A}$ \\
\hline $\begin{array}{l}\text { Knock-on electrons } \\
\text { from the foil }\end{array}$ & 1.2 & $\begin{array}{l}\text { up to } \\
2.4 \mathrm{MeV}\end{array}$ & $90 \mu \mathrm{A}$ \\
\hline Thermionic electrons & $<0.002$ & $\sim 0.24 \mathrm{eV}$ & $<0.12 \mu \mathrm{A}$ \\
\hline $\begin{array}{l}\text { Res. gas electron-ion } \\
\text { pairs }\end{array}$ & 0.0037 & $\begin{array}{l}\text { up to } \\
2.4 \mathrm{MeV}\end{array}$ & $0.28 \mu \mathrm{A}$ \\
\hline
\end{tabular}

PAC_25AP.DOC 42T/9S
$\mathrm{H}^{\circ}$ current is about $10 \mathrm{~mA}$ over the 250-ns chopping period, or about $7 \mathrm{~mA}$ averaged over the entire macropulse. In contrast to the other electron-production processes, there is no net charge deposited on the foil due to convoy-electron production. The kinetic energy of the convoy electrons is $430 \mathrm{keV}$.

The SEM coefficient for $800-\mathrm{MeV}$ protons incident on carbon is about 0.006 . Our foil has two surfaces, so we expect to see about 0.012 electrons leaving the surfaces of the foil for each proton that passes through the foil. During production conditions [1], the peak rate of SEM emission is about $0.12 \mathrm{~A}$, and the average rate (over a one second interval) is about $270 \mu \mathrm{A}$. The SEM electrons have kinetic energies up to about $20 \mathrm{eV}$.

To get the knock-on-electron production rate, we apply the equations of F. Sauli [2]: the number of electrons created with energy greater than $E_{0}$, in carbon of thickness $\rho t=200 \mu \mathrm{g} / \mathrm{cm}^{2}$, from a proton of velocity $\beta c$, is

$N=\left(0.154 \mathrm{MeV} \mathrm{cm}^{2} / \mathrm{g}\right) \cdot \frac{\rho t}{\beta^{2}} \cdot\left(\frac{1}{E_{0}}-\frac{1}{(2.45 \mathrm{MeV})}\right)$

We are interested in knock-on electrons with kinetic energies large enough to escape from the foil. If we we take the case of a knock-on electron created near the center of the foil, $E_{0}$ must be slightly greater than $5 \mathrm{keV}$ for the electron to escape. Using this value for $\mathrm{E}_{0}, N=0.004$. The rate of knock-on electron emission from the foil is therefore about one third of the SEM emission rate. The knock-on electrons have kinetic energies up to about $2.4 \mathrm{MeV}$. The probability of the electron having kinetic energy $\mathrm{E}$ is approximately proportional to $1 / \mathrm{E}^{2}$, so most of the electrons are concentrated at low kinetic energies.

To calculate the rate of electron emission due to thermionic emission, we note that the peak foil temperature is about $1900^{\circ} \mathrm{K}$ during production conditions. At this temperature the thermionic electron current density is $8.4 \mu \mathrm{A} / \mathrm{cm}^{2}$, and even if we assume the entire $16 \mathrm{~mm} \times 16 \mathrm{~mm}$ foil is heated this gives us peak current of just $22 \mu \mathrm{A}$, about four orders of magnitude less than the peak SEM current. The foil temperature does not change much over the 360 -ns period of the PSR. The thermionic current is highly sensitive to the foil temperature - for a foil temperature just $100^{\circ} \mathrm{K}$ higher, the current density will be $45 \mu \mathrm{A} / \mathrm{cm}^{2}$. The kinetic energies of these electrons is about $0.24 \mathrm{eV}$. 
To calculate the current we expect from residual-gas ionization, we use the simple formula [3] $I_{I O N}=n \sigma d I_{B E A M}$. In our case, for production conditions, the gas density $n=$ $9.7 \times 10^{15} / \mathrm{m}^{3}$, the ionization cross section $\sigma=94 \times 10^{-24} \mathrm{~m}^{2}$, the length of the ionization volume $d=4.5 \mathrm{~m}$, and the average current $l_{B E A M}=5.2 \mathrm{~A}$. Using these parameters, the average ionization current $I_{I O N}=21 \mu \mathrm{A}$. The various electron production rates can also be expressed in terms relative to the number of injected $\mathrm{H}^{0}$ particles, as shown in Table 1. The distribution of kinetic energies for these electrons is similar to the knock-on electrons - up to $2.4 \mathrm{MeV}$.

\section{E-FIELD AT THE FOIL SURFACE}

To find out what happens to electrons emitted from the foil, we need to calculate the E-fields. We start by simplifying the problem by assuming that the foil completely covers the beampipe aperture, that it is perfectly conducting, and that it is at zero potential. We also assume an electrostatic case, since the beam intensity does not vary much over the distance of several beam-pipe diameters, and since the magnetic field due to the beam is weak (for a $43 \mathrm{~A}$ beam passing through a circle of radius $0.5 \mathrm{~cm}$, the $B$-field at $0.5 \mathrm{~cm}$ is only $1.7 \times 10^{-3}$ Tesla). These assumptions simplify the problem enough to allow us to easily calculate the perpendicular component of the electric field at the surface of such a foil. The parallel component of the E-field is of course zero, since we are assuming the foil is perfectly conducting.

To calculate the E-field at the surface of the foil, we assume a uniform, centered distribution of charge of radius $b$, in a perfectly conducting beam pipe of radius $a$, that extends in the $z$ direction. The charge density $\rho$ is a function of $r$ and $z$ only. Due to the lack of space, we cannot go into more detail (see ref. 4 for more details), but we have solved [5] Poisson's equation to get the electric potential:

$$
\begin{gathered}
\phi_{\text {beam }}(r, z, d)=-\sum_{n=1}^{\infty} \frac{2 \rho a b J_{1}\left(j_{0 n} a / b\right) J_{0}\left(j_{0 n} r / b\right)}{\varepsilon_{0} j_{0 n}^{3} J_{1}\left(j_{0 n}\right)^{2} \sinh \left(j_{0 n} d / b\right)} \times \\
\left(\sinh \left(j_{0 n} z / a\right)-\sinh \left(j_{0 n}(z-d) / a\right)-\sinh \left(j_{0 n} d / a\right)\right.
\end{gathered}
$$

where $\varepsilon_{0}$ is the permittivity constant, $J_{0}$ and $J_{1}$ are Bessel functions, and $j_{0 n}$ is the $n^{\text {th }}$ zero of $J_{0}$. Figure 1 shows a plot of $\phi_{\text {beam }}(0, z, d)$, using realistic parameters from the PSR $(\rho=$ $2.2 \times 10^{-9} \mathrm{coul} / \mathrm{cm}^{3}, a=7.5 \mathrm{~cm}, b=0.5 \mathrm{~cm}, r=0, z=0, d=$ $50 \mathrm{~cm}$ [larger values of $d$ will not change the result]). We see that once we get about $25 \mathrm{~cm}$ away from the foil our potential has reached a maximum of $9800 \mathrm{~V}$, and that the potential climbs very steeply during the first couple of centimeters.

To get the E-field, we differentiate $\phi_{\text {beam }}$ to get

$$
E_{z}(r, z)=-\sum_{n=1}^{\infty} \frac{2 b \rho J_{0}\left(j_{0 n} r / a\right) J_{1}\left(j_{0 n} b / a\right) \sinh \left(j_{0 n}(z-d / 2) / a\right)}{\varepsilon_{0} j_{o n}^{2} J_{1}^{2}\left(j_{0 n}\right) \cosh \left(j_{0 n} d / 2 a\right)}
$$

From Fig. 1 we see that if the foil is biased with no beam present, low-energy electrons leaving the foil are pushed back onto the foil due to the triangle-shaped potential well. If the beam is present and the foil is grounded, low-energy electrons will be pulled off the foil due to the sharp drop in the potential well caused by the beam. If the beam is present and the foil is biased below the depth of the beam's potential, then we create a potential well a couple of centimeters from the surface of the foil. Low-energy electrons pulled off the foil will be trapped in this well until the proton bunch has gone by, and the electrons will then be pushed back onto the foil to be reabsorbed. 


Fig. 1 . A plot of the potential due to the beam (solid line),
the biased foil (dashed line) and the sum (bold). We assume
a 7.5 -cm-radius beam pipe with a full-aperture, perfectly
conducting foil at one end, a foil bias of $10,000 \mathrm{~V}$, and a
bea $\mathrm{m}$ radi us of 0.5 cm with a ch arge den sity of
$2.2 \mathrm{x} 10^{-9} \mathrm{C} / \mathrm{cm}^{3}$ (43 A). The dashed line does not contact
the $\mathrm{y}$ axis at $-10,000$ because of convergence problems at
$z=0$. The polarities of all the potentials have been reversed
to make the concept clearer.

\section{A RESISTIVE FOIL}

Of course the PSR stripper foil is not perfectly conducting. The 16-mm $\times 16-\mathrm{mm} \times 200 \mu \mathrm{g} / \mathrm{cm}^{2}$ carbon postage-stamp foil presently in use at the PSR is supported by about 115 ea. 5-micron-diameter carbon fibers, spaced at 2-mm intervals, and stretched across a rectangular metal frame with inside dimensions of $15.4 \mathrm{~cm} \times 17.0 \mathrm{~cm}$. The fibers form two planes of webbing that support the foil, and the average fiber length between the foil and the frame is about $7.5 \mathrm{~cm}$. We have measured the bulk resistivity of the fibers to be $1.2 \mathrm{~m} \Omega-\mathrm{cm}$, or $6 \mathrm{k} \Omega / \mathrm{cm}$. If we assume the fibers make good electrical contact with the frame and the foil, the total resistance is about $200 \Omega$. The carbon foil starts out as amorphous carbon with a very high bulk resistivity of about 4 or $5 \Omega-\mathrm{cm}$. It may anneal after spending some time in the beam, and therefore reduce the bulk resistivity, but this effect has not been studied. From all of this we see that the minimum resistance between the foil and the frame is about $200 \Omega$, and that it could be a lot higher due to marginal electrical contact between the foil and the fibers and between the fibers and the frame. To help minimize the resistance, in 1993 we started using conductive epoxy to fasten the fibers to the frame.

Because of the resistance between the foil and the frame, the potential of the foil will jump around as electrons are emitted from it. If the potential jumps are large enough, they can affect the electrons emitted from the foil. To estimate the magnitude of the jumps, we consider the case of a grounded foil frame, with no cable leading to any test equipment, with a perfectly-conducting foil sitting at the end of a $200 \Omega$ resistor. For a peak SEM and knock-on current of $0.16 \mathrm{~A}$, the potential of the foil should jump up by just $32 \mathrm{~V}$. This is not enough of a change in potential to significantly alter the electron trajectories. The time constant is less than $1 \mathrm{~ns}$, so the time structure of the potential fluctuations match the time structure of the beam.

\section{SUMMARY}

We have identified five different sources of electrons in the injection section of the PSR: convoy electrons, SEM electrons, knock-on electrons, thermionic electrons, and electrons from residual-gas ionization. We have characterized these sources with simple calculations of their energies and production rates. The three most prolific sources are the SEM, the knock-on. and the convoy electrons. We have also made some simple calculations of the potential and electric fields in the vicinity of a perfectly conducting, full-aperture, biased stripper foil with a beam passing through it. We found that by biasing the foil, we can create a potential well that will trap low-energy electrons emitted from the foil. This technique has been successfully applied [6] to control the electrons created in the injection section of the PSR.

This paper is just a beginning. More work is needed on modeling the E-field and potential distributions for a resistive foil, modeling the fields for an asymmetric gaussian beam distribution (as opposed to the uniform cylindrical distributions assumed in this note), and modeling the effect of a foil that covers only a fraction of the full aperture. More work is also needed to investigate sources of electrons other than those due to interactions of the beam with the stripper foil.

\section{ACKNOWLEDGMENTS}

I would like to acknowledge the help of $\mathrm{R}$. Cooper with solving Laplace's and Poisson's equations.

[1] Production conditions are characterized by an accumulation time of about $650 \mu \mathrm{s}$, a storage time of $10 \mu \mathrm{s}$, a rep rate of $20 \mathrm{~Hz}$, and an average current of about $75 \mu \mathrm{A}$ of $800-\mathrm{MeV}$ protons delivered to the spallation target.

[2] F. Sauli, "Principles of Operation of Multiwire Proportional and Drift Chambers," CERN 77-09, May 3, 1977.

[3] G Guinard, "Selection of Formulae Concerning Proton Storage Rings", CERN 77-10, June 6, 1977.

[4] M. Plum, "Electric Fields, Electron Production, and Electron Motion at the PSR Stripper Foil", PSR Tech Note PSR-94-001, March 10, 1994.

[5] R. Cooper, private communication.

[6] M. Plum et. al., "Electron Clearing in the Los Alamos Proton Storage Ring", 1995 IEEE Particle Accelerator Conference, these proceedings. 